\title{
Role of proteolytic enzymes in human prostate bone metastasis formation: in vivo and in vitro studies
}

\section{CA Hart*,', LJ Scott', S Bagley', AAG Bryden ${ }^{2,3}$, NW Clarke ${ }^{2,3}$ and SH Lang ${ }^{4}$}

'Cancer Research UK - Group of Experimental Haematology, Paterson Institute for Cancer Research, Christie Hospital NHS Trust, Wilmslow Road, Manchester M20 4BX, UK; ${ }^{2}$ Department of Urology, Salford Royal Hospital, Eccles Old Road, Salford, Manchester, M6 8HD, UK; ${ }^{3}$ Christie Hospital NHS Trust, Wilmslow Road, Manchester M20 4BX, UK; ${ }^{4}$ YCR Cancer Research Unit, Biology Department, The University of York, York YOIO 5YW, UK

Prostate cancers ability to invade and grow in bone marrow stroma is thought to be due in part to degradative enzymes. The formation of prostate skeletal metastases have been reproduced in vitro by growing co-cultures of prostatic epithelial cells in bone marrow stroma. Expression of urokinase plasminogen activator, matrix metalloproteinase I and 7 by prostatic epithelial cells were identified using immunocytochemistry. Also, in vivo tissue sections from human prostatic bone marrow metastases were stained. To establish the role of these enzymes on colony formation, inhibitory antibodies directed against urokinase plasminogen activator, matrix metalloproteinase I and matrix metalloproteinase 7 were added into primary prostatic epithelial cells and bone marrow stroma co-cultures. All prostatic epithelial cell cultures stained positively for matrix metalloproteinase I, matrix metalloproteinase 7 and urokinase plasminogen activator. Generally prostatic epithelial cells derived from malignant tissues showed increased staining in comparison to epithelia derived from non-malignant tissue. In agreement with in vitro cocultures, the in vivo tissue sections of prostate bone marrow metastases showed positive staining for all three enzymes. Inhibition studies demonstrated that blocking matrix metalloproteinase I, matrix metalloproteinase 7 and urokinase plasminogen activator function reduced the median epithelial colony area significantly in bone marrow stroma co-cultures in vitro. Using a human ex-vivo model we have shown that matrix metalloproteinase I, matrix metalloproteinase 7 and urokinase plasminogen activator play an important role in the establishment of prostatic epithelial cells within bone marrow. British Journal of Cancer (2002) 86, I|36-||42. DOI: 10.1038/sj/bjc/6600207 www.bjcancer.com (C) 2002 Cancer Research UK

Keywords: prostate cancer; prostate epithelia; bone marrow stroma; metastasis; urokinase plasminogen activator; matrix metalloproteinase

Prostate cancer $(\mathrm{CaP})$ is the second commonest cancer in males in the UK with 9500 deaths in 1999 (CRC, 2001/2002) and commonly metastasises to the bone marrow (Jacobs, 1983). Fifty per cent of patients who present clinically with $\mathrm{CaP}$ already have incurable bone marrow metastases (George, 1988) and morbidity and death is usually a direct consequence of these. Therefore it is of importance that the mechanisms involved in the metastatic process be understood.

Tumour cells need to penetrate the extra-cellular matrix (ECM) and basement membrane in order to metastasise. The major components of the ECM are collagens (types I-V), proteoglycans, elastin, laminin and fibronectin, and in order to break free from the primary site and invade at the secondary site, tumour cells must overcome these structural barriers (Woolley, 1993). In recent years the interactions of various proteases have been identified in this crucial step, including the matrix metalloproteinase (MMP) and the serine protease family (plasmin and urokinase plasminogen activator (uPA)). The relative contributions of these proteases in tumour cell invasion has been in debate. More recent data have implicated the MMP family in a more complex role than ECM degradation alone. They have also been found to be actively

*Correspondence: CA Hart; E-mail: chart@picr.man.ac.uk Received II January 200 I; revised I 3 November 200 I; accepted 2 I January 2002 involved with generating factors that can stimulate tumour cell (Stetler-Stevenson and $\mathrm{Yu}, 2001$ ) and endothelial migration and angiogenesis needed for tumour formation (John and Tuszynski, 2001). The matrix metalloproteinase family comprises at least 20 zinc dependent endopeptidases clearly identified, with more (up to MMP-26) currently being characterised (Marchenko et al, 2001). Normally they are associated with programmed cellular events such as tissue remodelling, wound healing, uterine and breast involution and organogenesis in development (Chambers and Matrisian, 1997). Originally members of the MMP family were thought to be substrate specific. However, it has been recognised that each can degrade multiple substrates (John and Tuszynski, 2001).

In this study we looked at specific proteases that could be active in $\mathrm{CaP}$ and its secondary metastatic site, the bone marrow. MMP-7 (matrilysin) exhibits high activity against collagen IV, a major component of bone marrow stroma (BMS) and other basement membranes and has been found previously in carcinoma cells of many cancers including prostate and their metastases (Pajouh et al, 1991). In contrast, MMP-1 (interstitial collagenase) has a high substrate affinity for collagen I, also a major component of BMS (Takahashi et al, 1994). MMP-1 is thought to be expressed mainly by fibroblasts/stromal cells and is involved with interactions between the tumour cells and host fibroblasts in the remodelling of the ECM (Heppner et al, 1996). MMP-1 production has also been found to be up-regulated during cell-cell contact with breast 
carcinoma cells and bone marrow fibroblasts (Saad et al, 2000). Urokinase plasminogen activator is also found in human primary prostate carcinomas and in bone metastases (Kirchheimer et al, 1985). Data obtained from a limited number of cell lines have indicated that uPA is associated with the more aggressive type of carcinoma and facilitates invasion (Hoosein et al, 1991; Festuccia et al, 1998). These proteases are therefore of particular interest in the metastatic development of prostate cancer.

As yet, no human model has been established to study variations in expression and effects of MMP-1, MMP-7 and uPA on the formation of prostate bone metastases in vitro. The prostatic epithelial cell (PEC)/BMS co-culture system as described by Lang et al (1998) represents an ideal in vitro model for this purpose. Here, we propose that enzyme mediated cell interactions maybe important for the expansion of epithelial colonies within BMS.

\section{MATERIALS AND METHODS}

\section{Materials}

All reagents were purchased from Sigma-Aldridge, Poole, UK. All tissue culture medium and horse serum was from Life Technologies, Paisley, UK with the exception of Ham's F12 media, PAA Laboratories, Austria. Foetal calf serum (FCS) was supplied by Sera Labs, Sussex, UK and Worthington collagenase type 1 and trypsin from Lorne Laboratories Ltd., Twyford, UK.

\section{Antibodies}

MMP-7 and uPA were from Chemicon International, Harrow, UK; MMP-1 and mouse IgG1 from R\&D Systems, Abingdon, UK; mouse anti-human pan cytokeratin from Sigma-Aldridge, Poole, UK; rabbit anti-human pan cytokeratin from Biogenesis Ltd., Poole, UK; rabbit anti mouse biotinylated antibody, swine antirabbit TRITC and aqueous mounting medium from DAKO Ltd., Cambridge, UK. Vectastain Elite ABC kit from Vector Laboratories, CA, USA and streptavidin Alexa Fluor ${ }^{\mathrm{R}} 488$ was from Molecular Probes, Oregon, USA.

\section{Cell lines}

The malignant prostate cell line PC-3 (bone marrow metastasis derived) (Kaighn et al, 1979) and non-malignant line PNT2-C2 (Berthon et al, 1995) were cultured in Ham's F12, 7\% FCS and $2 \mathrm{mM}$ L-glutamine and in RPMI 1640, 10\% FCS and $2 \mathrm{mM} \mathrm{L}$ glutamine respectively. Cultures were grown at $37^{\circ} \mathrm{C}$ in a humidified atmosphere of $5 \% \mathrm{CO}_{2}$ in air.

\section{Primary tissue collection and culture}

With informed consent prostatic tissue was obtained from males undergoing treatment for malignant $(\mathrm{CaP})$ or non-malignant benign prostatic hyperplasia (BPH) disease. Each individual prostate chip was bisected with half undergoing histological analysis for diagnostic evaluation and the remainder used for tissue culture. Prostate epithelia and fibroblasts were isolated by collagenase digestion followed by differential centrifugation as described (Lang et al, 1998). Epithelial cells were initially grown in flasks and then used at passage $1-3$.

Human ribs removed for access during routine renal surgery were prepared for tissue culture using the method of Coutinho et al (1993). Bone marrow cells were flushed out from the rib and were resuspended in long-term culture medium (Iscove's Modified Dulbecco's Medium containing 10\% FCS, 10\% horse serum and $5 \times 10^{-7} \mathrm{M}$ hydrocortisone) and $2 \times 10^{7}$ cells plated into $25 \mathrm{~cm}^{2}$ tissue culture flasks. The cultures were grown at $33^{\circ} \mathrm{C}$ in $5 \% \mathrm{CO}_{2}$ in air for 4-5 weeks until haemopoietically active areas were observed.

\section{Co-culture experiments}

Bone marrow stromal cultures were trypsinised (0.5\% trypsin) and re-seeded into 24 -well culture plates (or 4-well glass slide flask) at the same cell density and incubated for $48 \mathrm{~h}$.

The media on the BMS was removed and replaced with longterm culture medium plus Keratinocyte-SFM (1:3) containing 500 epithelial cells (PC-3, PNT2-C2 or primary PEC) per well. The co-cultures were incubated for $7-10$ days at $37^{\circ} \mathrm{C}$ then fixed with methanol/acetone $(1: 1)$, allowed to dry and frozen at $-20^{\circ} \mathrm{C}$ until required.

\section{Immunocytochemistry}

Samples were incubated with primary antibodies overnight at $4{ }^{\circ} \mathrm{C}$ in a humidified chamber after blocking with $10 \%$ serum then $0.3 \%$ hydrogen peroxide. Anti-MMP-7, anti-uPA, anti-MMP-1 and mouse IgG1 were prepared at $20 \mu \mathrm{g} \mathrm{ml}^{-1}$ and mouse anti-human pan cytokeratin at $1: 200$. This was followed by addition of biotinylated rabbit anti mouse $1: 400$ for $40 \mathrm{~min}$ at room temperature. A complex of avidin $\mathrm{DH}$ and biotinylated horseradish peroxidase $\mathrm{H}$ (Vectastain Elite $\mathrm{ABC}$ kit) was then added for 15-20 min, followed by the addition of DAB substrate for 5-10 min. After washing with distilled water the cells were counterstained using haematoxylin and fixed in aqueous mounting fluid. Samples were scored as to the intensity of brown staining: $(-)$ blue haematoxylin counterstain visible only, $(-/+)$ pale brown, $(+)$ brown, $(++)$ dark brown. These staining intensities were set using agreed criteria and results were corroborated with more than one investigator.

For dual fluorescent staining, cultures were fixed, blocked and labelled for primary antibodies as observed above. Rabbit antihuman cytokeratin $(1: 100)$ was added for $30 \mathrm{~min}$, followed by a swine anti-rabbit TRITC $(1: 20)$ for $1 \mathrm{~h}$ in the dark. The samples were blocked for $30 \mathrm{~min}$ using $\mathrm{PBS} / 10 \%$ rabbit serum and $1 \%$ BSA, then incubated with rabbit anti-mouse biotinylated antibody $(1: 400)$ for $30 \mathrm{~min}$. After washing, streptavidin Alexa Fluor ${ }^{\mathbb{B}} 488$ $\left(5 \mu \mathrm{g} \mathrm{ml}^{-1}\right)$ was added for $1 \mathrm{~h}$ in the dark.

Cells were observed using an Olympus BX51 upright microscope, with a Plan-Apochromat $40 \times$ objective (NA 1.0, oil immersion). Using a triple-band bypass filter (Chroma Technology Corp. set: 61000 v2SBX for DAPI/FITC/TRITC) the interaction of TRITC/Cytokeratin (ex.542 nm em.560 nm), FITC/uPA, MMP-1, MMP-7 (ex.488 nm em.505-530 nm) and DAPI (ex.360 em.460) were examined. Images were captured via a cooled Colourview 12 and the analySIS imaging acquisition and processing system (SiS, GmbH). Images were captured at $1300 \times 1030,24$ bit resolution with an exposure time of $5 \mathrm{~s}$. Processing and montaging of images was then carried out using Adobe PhotoShop 6.0 (Adobe Systems Inc.)

\section{Immunohistochemistry}

Prostatic bone marrow metastases were sectioned from $8 \mathrm{~mm}$ trephine core biopsies taken from the iliac crest of patients with untreated prostate cancer. The undecalcified paraffin embedded sections were first de-waxed in two changes of xylene for $5 \mathrm{~min}$, then run through a series of alcohols to rehydrate the sections. Sections were immersed in citrate solution $\mathrm{pH} 6.0$ and placed in a microwave on full power for $25 \mathrm{~min}$. The solution was allowed to cool and was exchanged for water. Samples were stained and scored according to protocols shown above.

\section{Inhibition studies}

Primary epithelial cell/BMS co-cultures were set up as above, in addition to which the epithelial cells were initially allowed to bind to the BMS for $4 \mathrm{~h}$ at $37^{\circ} \mathrm{C}$ before antibodies against either uPA, MMP-1 and MMP-7 (functionally blocking) were added at $2 \mu \mathrm{g}$ 
well $^{-1}$ daily. Mouse IgG1 was added to the control well. After 7 days at $37^{\circ} \mathrm{C}$ the cultures were fixed using methanol/acetone $(1: 1)$, dried then immunostained for cytokeratin. Colony areas were measured using an eyepiece graticule after calibration with a slide graticule. For each well $>100$ colonies were measured for length and width. The median colony area per well was then calculated and statistical analysis achieved using Wilcoxon's Rank Sum Test.

\section{RESULTS}

\section{Epithelial colony morphology and enzyme characterisation in BMS co-culture}

The cell lines PC-3, PNT2-C2 (derived from normal prostate), and primary epithelial cells (derived from patients with $\mathrm{CaP}$ or $\mathrm{BPH}$ ) were initially grown on BMS and stained for cytokeratin to observe colony morphologies. As a comparison to the malignant cell line PC-3, the PNT2-C2 cell line was used to represent normal prostate cell behaviour. We also used primary PEC derived from patients with $\mathrm{CaP}$ and compared them to $\mathrm{BPH}$ derived epithelial cells. Figure 1 (CK) shows the appearances of the different epithelial cells grown on BMS and labelled with cytokeratin. Primary epithelial cells derived from patients with $\mathrm{CaP}$ grew colonies that appeared either spiky or round and compact. Epithelial cells derived from patients with $\mathrm{BPH}$ always formed round and compact colonies. Retraction of the BMS was also seen with cells derived from $\mathrm{CaP}$. Cell lines showed dispersed PC-3 colonies but compact PNT2-C2 colonies.
Co-cultures were also dual-stained for cytokeratin, and either MMP-1, MMP-7 or uPA to establish that prostate epithelial colonies produce these enzymes in BMS. Epithelial cells derived from $\mathrm{CaP}$ stained positively for MMP-1 and MMP-7 when grown in BMS co-culture (Figure 1). Some of the bone marrow stroma at the interface with the PEC (orange/yellow) had retracted away and showed intense MMP-1 green staining (arrows). Urokinase plasminogen activator staining was positive but less intense. Colonies from BPH derived epithelial cells showed staining of MMP-1, MMP-7 and uPA located within the PEC with less marked staining towards the edge of the colony.

Co-cultures of the cell lines showed that all three enzymes were observed throughout the PC-3 cells. This co-localised with cytokeratin at the cell membrane/pseudopodial processes. The PNT2-C2 cell line showed some weak cytoplasmic staining for all the enzymes, but they were predominantly cytokeratin positive.

To compare the presence of MMP-1, MMP-7 or uPA when epithelial cells (cell lines and primaries) were grown on tissue culture plastic or normal human BMS, cultures were immuno stained, using DAB. The results summarised in Table 1 showed that the PC-3 cell line expressed strong staining for all the enzymes tested on plastic, except MMP-1, but this was up-regulated in the presence of BMS. The non-malignant cell line PNT2-C2 demonstrated absent or low staining intensities for all the enzymes on plastic but showed up-regulation of MMP-1 and UPA on BMS, with uPA staining the most. Primary PEC cultures from patients with $\mathrm{CaP}$ showed positive staining for all the enzymes on plastic, which was more intense than in the cells derived from BPH. There was no difference in staining between $\mathrm{CaP}$ and $\mathrm{BPH}$ on BMS for
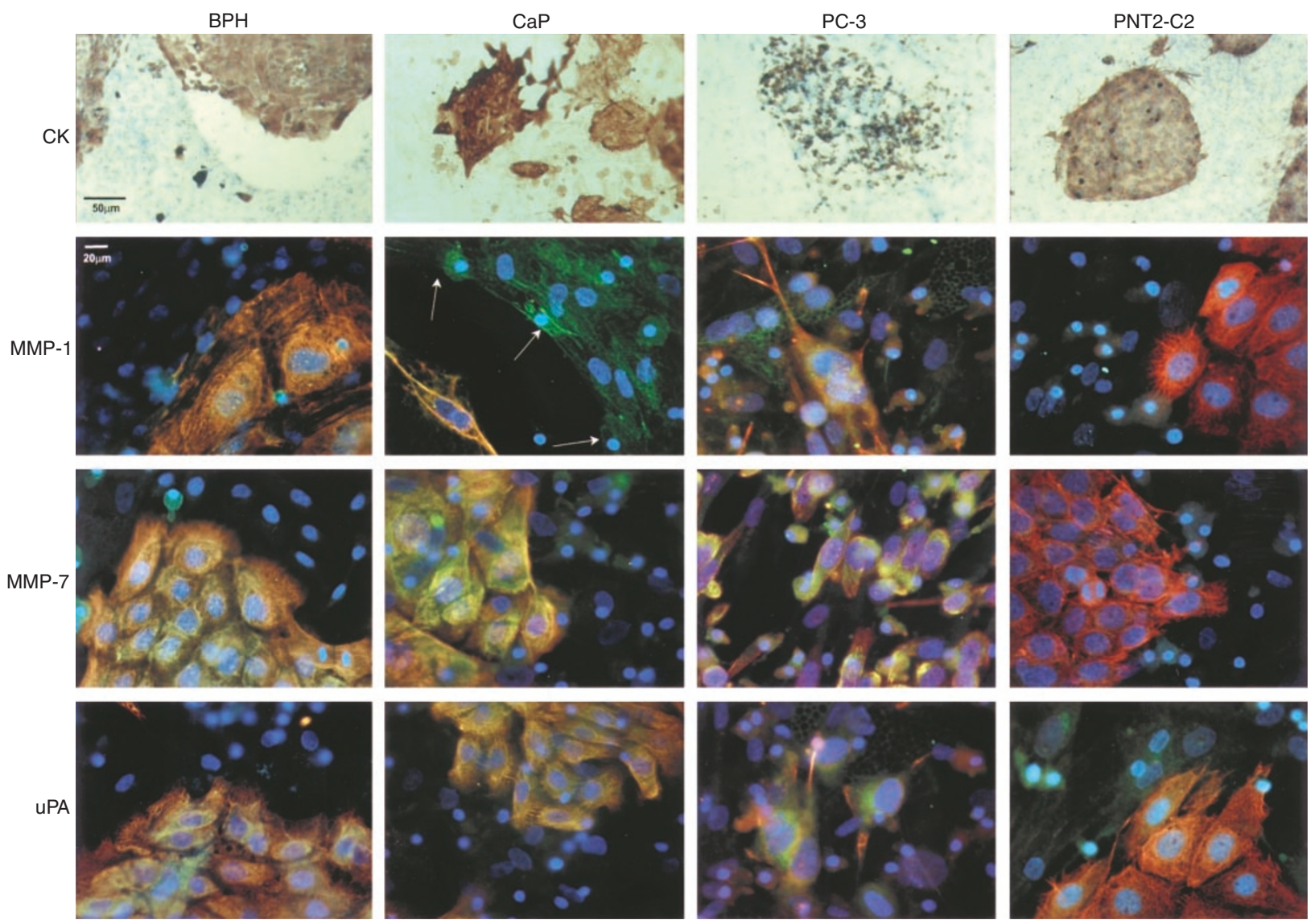

Figure I Enzyme characterisation of primary prostate epithelial cells and the prostate cell lines grown with BMS. Immunocytochemical staining showing typical colonies of cytokeratin (CK) positive (DAB brown staining) and fluorescent dual staining of epithelial cells grown in BMS co-culture (each picture is unrelated). Enzyme stained photographs are shown as dual fluorescence: red=cytokeratin; green=location of either uPA, MMP- I or MMP-7; yellow=double staining; blue=DAPI nuclear counterstain $(n=5)$. All negative controls were black. Arrows indicate retracted stromal edge. 
MMP-7 and uPA. However, MMP-1 expression was greater in CaP derived cells compared to BPH. These results were consistent in several primary and cell line cultures.

\section{Enzyme characterisation of bone marrow metastases}

Using bone marrow metastasis sections from patients with $\mathrm{CaP}$ it was possible to compare the staining pattern of the enzymes in vivo to the in vitro model. These sections were previously identified as prostatic bone marrow metastases by positive PSA staining (Bryden et al, 2002). Figure 2 shows immunohistochemical (DAB) staining for MMP-1, MMP-7 and uPA using the same concentration of antibody as for the co-culture staining. Serial sectioning allowed comparison of the different enzyme staining patterns within the same area of tissue. Areas of cytokeratin positive epithelial cells

Table I Immunocytochemical (DAB) staining for the proteolytic enzymes in prostate epithelial cells derived from patients with either $\mathrm{CaP}$ or $\mathrm{BPH}$ and in the PC-3 and PNT2-C2 cell lines grown either on plastic or together with BMS as a co-culture

\begin{tabular}{lcccccc}
\hline & \multicolumn{2}{c}{ MMP-I } & \multicolumn{2}{c}{ MMP-7 } & \multicolumn{2}{c}{ uPA } \\
& Plastic & BMS & Plastic & BMS & Plastic & BMS \\
\hline PC-3 & + & ++ & ++ & ++ & ++ & ++ \\
PNT2-C2 & - & $-/+$ & $-/+$ & $-/+$ & $-/+$ & + \\
CaP & $+/++$ & + & + & + & + & + \\
BPH & + & $-/+$ & $-/+$ & + & $-/+$ & + \\
\hline
\end{tabular}

The staining intensity is equivalent to $-=$ negative, $-1+=$ weak positive (light brown), $+=$ positive (brown), $++=$ strong positive (dark brown). $\mathrm{CaP} n=4, \mathrm{BPH} n=3$ (median score).

A
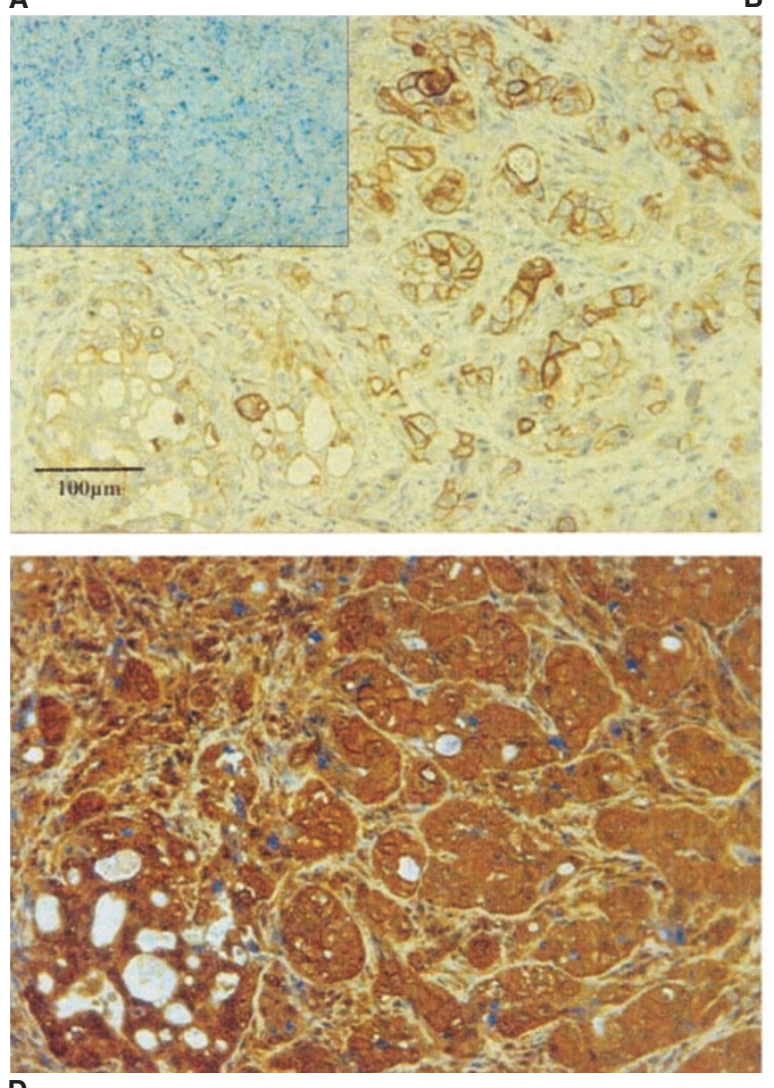

within the bone marrow structure can be seen in Figure 2B. The epithelial colonies showed high expression of MMP-1 (C) and MMP-7 (D), with less intense staining in the surrounding stroma. Figure 2E showed weaker staining for uPA than was observed for MMP staining, but again this was more clearly stained in the epithelial region than the stromal area.

Prostate bone metastases $(n=7)$ were sectioned and stained for cytokeratin, MMP-1, MMP-7 and uPA. Median staining scores were then calculated and summarised in Table 2. One sample was cytokeratin negative but was identical in morphology to the cytokeratin positive epithelia in the other samples. Generally samples stained strongest for MMP-1, then MMP-7 and more weakly for uPA. The staining intensity was strongest towards the outer edges of the epithelial cells.

Table 2 Enzyme immunohistochemical (DAB) staining of prostatic bone marrow metastasis sections

\begin{tabular}{lcccc}
\hline Sample & MMP-I & MMP-7 & uPA & Cytokeratin \\
\hline 1 & ++ & ++ & $-/+$ & $-/+$ \\
2 & ++ & ++ & $-/+$ & ++ \\
3 & + & + & $-/+$ & ++ \\
4 & ++ & + & $-/+$ & ++ \\
5 & + & $-/+$ & - & + \\
6 & + & $-/+$ & $-/+$ & ++ \\
7 & ++ & + & $-/+$ & - \\
Median & ++ & + & $-/+$ & ++ \\
\hline
\end{tabular}

Samples of seven undecalcified prostatic bone metastases were cut and labelled with the antibodies shown. Because of patient heterogeneity, the median staining score is given at the bottom of the table. - =negative staining; $-1+=$ weak positive (light brown); +=positive (brown); ++=strong positive (dark brown).

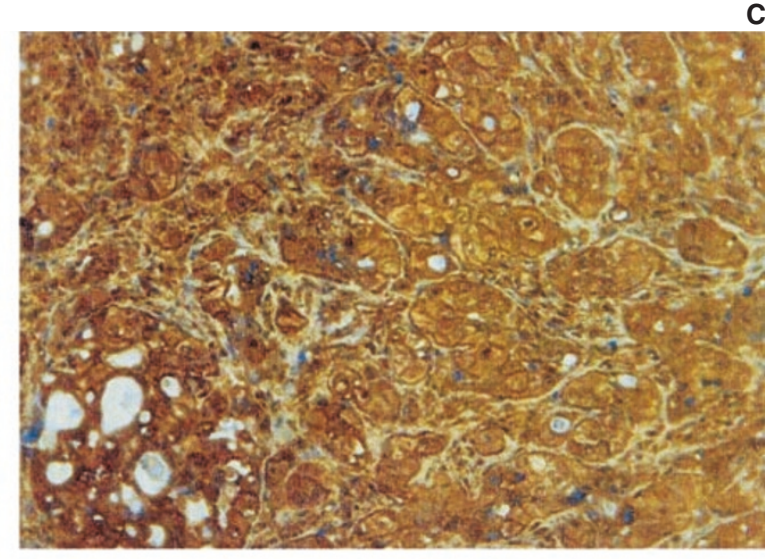

\section{C}

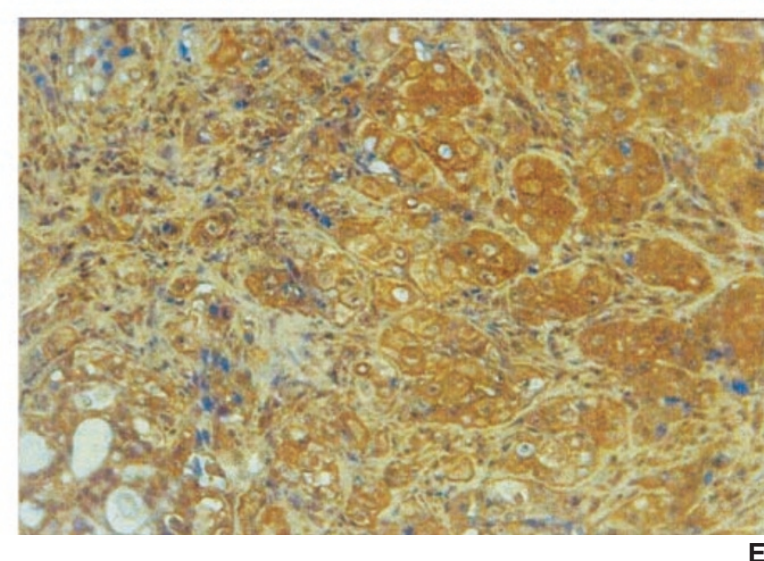

Figure 2 Serial sections of a prostatic bone marrow metastasis. Typical examples of the enzyme staining found from seven samples (see Table 2). Each photograph shows the same region of sample immunohistochemically (DAB) labelled for: (A) negative control (haematoxylin), (B) cytokeratin indicating the colonies of epithelial cells within the bone marrow space, (C) MMP-I, (D) MMP-7 and (E) UPA. 


\section{Inhibition studies}

Functionally blocking antibodies against the proteolytic enzymes were added to the in vitro co-culture system to further study the role of these enzymes in epithelial colony formation. As the enzymes are secreted continually by the epithelial or stromal cells, antibodies were added daily. Titration of the antibodies indicated that $2 \mu \mathrm{g} \mathrm{ml}^{-1}$ consistently and significantly inhibited growth of epithelial colonies (data not shown). Figure 3A shows the appearance of typical BPH derived colonies after treatment with the antibodies, cultures with samples derived from patients with $\mathrm{CaP}$ showed a similar appearance. The control co-culture showed large colonies, whereas the co-cultures treated with the blocking antibodies showed smaller irregularly shaped colonies. Results were similar whether epithelial cells were derived from benign or malignant tissue. This experiment was repeated on primary epithelial cell samples, derived from malignant $(n=6)$ or benign tissue $(n=7)$ shown in Figure 3B,C. Patient samples varied in response to these blocking antibodies, but overall, samples derived from patients with $\mathrm{CaP}$ and $\mathrm{BPH}$ showed greatest reduction in colony area with anti-MMP-7, followed by anti-MMP-1 then anti-uPA. Median per cent reduction of colony area for $\mathrm{CaP}$ derived colonies compared to the control was 48,45 and $23 \%$, with $\mathrm{BPH}$ derived colonies 48,45 and $37 \%$ respectively. There was no significant difference observed between the responses for $\mathrm{CaP}$ or $\mathrm{BPH}$ derived epithelial cells. However, statistical analysis showed significantly reduced $(P<0.05)$ colony growth after anti-MMP-1 was added to the culture in $6 / 6 \mathrm{CaP}$ samples and $6 / 7 \mathrm{BPH}$ samples. Inhibiting MMP-7 significantly decreased $(P<0.05)$ colony size in $5 / 6 \mathrm{CaP}$ and $7 / 7 \mathrm{BPH}$ samples and blocking uPA activity significantly reduced $(P<0.05)$ growth in $1 / 3 \mathrm{CaP}$ and $4 / 4 \mathrm{BPH}$ samples (Wilcoxon's Rank Sum Test) compared to the control.

The total numbers of colonies formed in the presence of the antibodies was variable, either remaining the same or increasing above that of the control (data not shown).

\section{DISCUSSION}

Prostate cancer shows a predilection to metastasise to the bone marrow. We have used in vitro models (Lang et al, 1998) to identify potential enzymes involved in this process and compared these with in vivo effects in human bone marrow metastasis sections.

Initially we looked at the colony morphology of PEC when grown in BMS. The PC-3 cell line and cells derived from patients with $\mathrm{CaP}$ showed colonies and cells which appeared spiky and scattered whilst the PNT2-C2 cell line and cells derived from patients with $\mathrm{BPH}$ showed rounded colonies. This confirmed findings previously reported (Lang et al, 2000) which suggests a more motile and invasive nature of PC-3 and CaP derived epithelial cells. Regarding proteolytic enzyme production in BMS co-culture, we found that when cells derived from patients with $\mathrm{CaP}$ were grown in BMS co-culture, not only were the epithelial cells strongly stained for MMP-1, the stromal cells around the leading edge of the colony were also strongly positive. This has been shown in previous studies where in vivo MMP-1 expression was found in the stromal area along the invasive margin in gastric cancer (Migita et al, 1999) and breast cancer (Heppner et al, 1996). Also, in vitro MMP-1 expression was up-regulated by cell-cell contact between BMS and breast carcinoma cells (Saad et al, 2000). This may indicate subtle tumour/stroma interactions causing increased secretion of these enzymes. Epithelial cells derived from patients with BPH showed enzyme staining similar to $\mathrm{CaP}$ derived cells but less was seen at the stromal margins of these colonies, suggesting no stimulation of the stromal cells to secrete these enzymes. Clearly interactions between BMS and epithelial cells play an important role in the level of enzyme expression.

Using immunocytochemistry we compared the enzyme levels between the cell lines PC-3, PNT2-C2 and the primary PEC.
Previous studies showed that the PC-3 cell line expresses uPA (Hoosein et al, 1991) and mRNA for 10 MMPs including MMP1 and MMP-7 (Giambernardi et al, 1998). We confirmed that the PC-3 cell line gave strong positive staining for uPA, MMP-1 and MMP-7. By comparison, the PNT2-C2 cell line gave weak staining for these enzymes, which was marginally increased in BMS co-cultures. For the primary PEC, median staining values were shown due to patient variability. This phenomenon must not be overlooked as differing enzyme expression between patients could provide an insight into why prostate cancer can be highly variable in its disease progression. Generally, primary samples from patients with $\mathrm{CaP}$ stained more strongly for these enzymes than samples derived from $\mathrm{BPH}$ when grown on tissue culture plastic. However, apart from MMP-1 (increased staining for $\mathrm{CaP}$ in BMS compared to BPH cells) we did not find a difference between the staining of $\mathrm{CaP}$ or $\mathrm{BPH}$ derived samples when grown in the BMS co-culture. In support of this, it has been reported that both $\mathrm{CaP}$ and $\mathrm{BPH}$ epithelial cells will grow and invade in BMS coculture in a similar way (Lang et al, 1998), despite the fact that although BPH cells do not invade the bone marrow in vivo they are released into the circulation during routine TURP for bladder outflow obstruction (McIntyre et al, 2000, 2001). Therefore, subtle differences in enzyme expression between $\mathrm{CaP}$ and $\mathrm{BPH}$ epithelial cells could make a difference to these circulating cells in determining their ability to invade through endothelium and ultimately grow in the bone marrow itself.

Our results indicated very subtle differences in enzyme expression between the $\mathrm{CaP}$ and $\mathrm{BPH}$ primary epithelial cells when grown in BMS whereas in previous in vivo studies greater differences were observed. Hashimoto et al (1998) demonstrated a significant correlation between the levels of MMP-7 mRNA in the primary prostate carcinoma and pathological stage and mean serum levels of uPA were reported to be significantly higher in patients with $\mathrm{CaP}$ compared to healthy individuals or patients with BPH (Miyake et al, 1999). However, no significant correlation was made in a study carried out to identify changes in blood plasma levels of MMP-1 between patients with $\mathrm{CaP}$ or with $\mathrm{BPH}$ (Lein et al, 1998).

Our in vivo staining of prostate bone marrow metastases from patients with $\mathrm{CaP}$ showed positive staining for MMP-1 and MMP-7 but less intense staining for uPA. Previous work has demonstrated that MMP-1 levels in uncultured primary prostate carcinoma are very low and variable (Varani et al, 2001), whereas we have demonstrated that MMP-1 is strongly expressed in the bone metastases of prostate cancer patients. Our in vitro data also showed that in BMS co-culture, MMP-1 staining was greater in $\mathrm{CaP}$ co-cultures compared to $\mathrm{BPH}$ co-cultures. This reiterates the proposition that up-regulation of MMP-1 secretion maybe an important factor in the formation of prostate bone marrow metastases. As these changes in enzyme expression were subtle, further work using zymography may clarify these results and give quantitative data to corroborate our qualitative data.

Until now there has been no human in vitro model to demonstrate the role of proteolytic enzymes on metastasis formation, and there have been few studies to determine whether blocking these enzymes causes significant effect (although, previous animal models have shown that rats inoculated with Dunning R 3227, Mat LyLu rat prostate carcinoma cell line transfected to over-express uPA developed hind limb paralysis much faster than transfectants which under-expressed uPA (Achbarou et al, 1994)).

The BMS co-culture system was used as a human ex-vivo model to study the effects of blocking enzyme activity on prostate epithelial colony growth enabling direct observation of their importance in the process of invasion. In most samples total median colony area was reduced by around 50\% following inhibition of MMP-1 and MMP-7. For uPA the reduction was less but still significant. In this study, cells from the primary tumour site rather than cells from metastatic bone biopsies were grown. Kirchheimer et al 

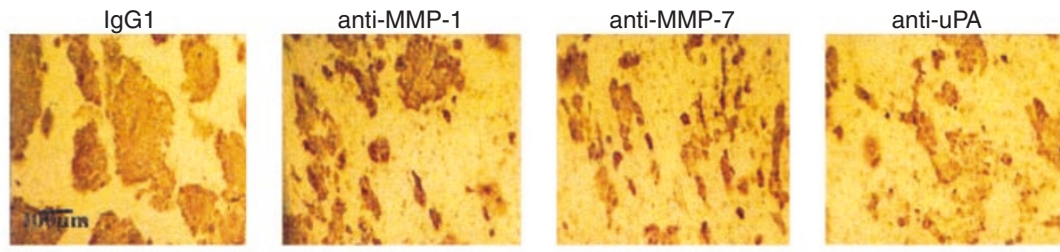

B CaP

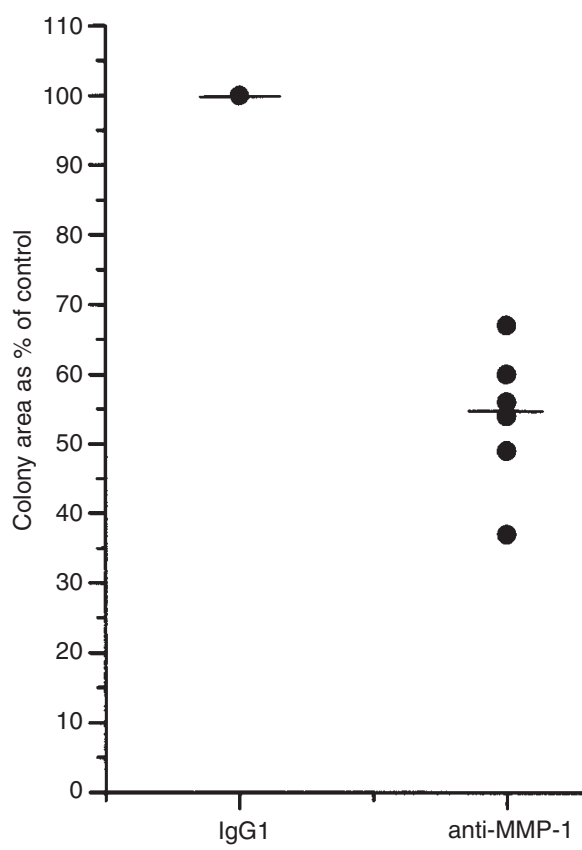

$\mathrm{C}$ BPH

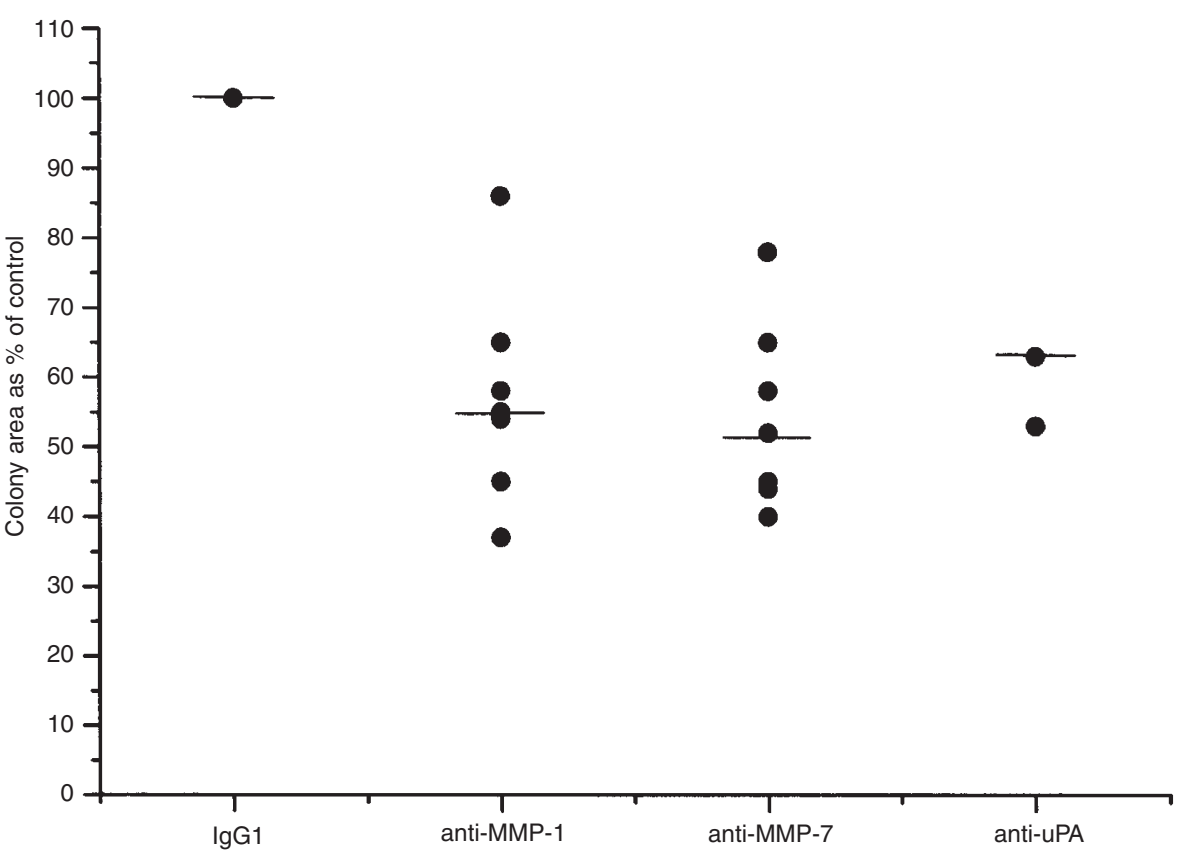

Figure 3 Antibody inhibition of epithelial colony area (\%). A comparison of the inhibition of prostate epithelial colony area in the presence of enzyme antibodies was made for a variety of primary epithelial samples. Typical immunocytochemical (DAB) cytokeratin staining of a BPH epithelial cell/BMS coculture in the presence of non-specific mouse IgGI, anti-MMP-I, anti-MMP-7 and anti-uPA antibodies ( $2 \mu g_{\text {day }}{ }^{-1}$ ) after 7 days in culture is shown in $(\mathbf{A})$. Median area of $>100$ colonies per sample were calculated and inhibition was calculated as a per cent of the control area. Median percentage inhibition is represented by the bar: (B) CaP, MMP-I and MMP-7 $(n=6), \mathrm{uPA} n=4 ;(\mathbf{C}) \mathrm{BPH}, \mathrm{MMP}-\mathrm{I}$ and MMP-7 $(n=7), \mathrm{uPA}(n=3)$. 
(1985) showed that out of 14 primary prostatic carcinomas and their respective bone metastases, the bone metastases had 1.5 times higher uPA than the corresponding primary tumour. For future study it would be interesting to ascertain whether greater reduction in colony size could be achieved by using prostate bone metastatic cells in the BMS co-culture.

Blocking MMP enzyme function caused reduction in colony size but not their number. Therefore, initial establishment of epithelial colonies within BMS was not affected although the subsequent growth and formation of these colonies was. Recent evidence has revealed that the MMPs not only cause degradation of the ECM but also interact with adhesion molecules and can stimulate cell migration and invasion. What we observed may have been therefore the result of cell migration and colony formation failure due to the neutralising effect of the antibodies (Stetler-Stevenson and Yu, 2001).

In summary, we have used the in vitro BMS co-culture system to show that MMP-1, MMP-7 and uPA are expressed by the bone metastatic prostate cell line $\mathrm{PC}-3$, primary $\mathrm{CaP}$ and $\mathrm{BPH}$ derived epithelial cells. They are also up-regulated in the non-malignant

\section{REFERENCES}

Achbarou A, Kaiser S, Tremblay G, Ste Marie LG, Brodt P, Goltzman D, Rabbani SA (1994) Urokinase overproduction results in increased skeletal metastasis by prostate cancer cells in vivo. Cancer Res 54: 2372 2377

Berthon P, Cussenot O, Hopwood L, Le Luc A, Maitland NJ (1995) Functional expression of SV40 in normal human prostatic epithelial and fibroblastic cells: Differentiation pattern of non-tumourigenic cell lines. Int J Oncol 6: $333-343$

Bryden AAG, Islam S, Freemont AJ, Shanks JH, George NJ, Clarke NW (2002) Parathyroid hormone-related peptide: expression in prostate cancer bone metastases. Prostate Cancer and Prostatic Diseases. Br J Cancer 86: $322-325$

CRC - Cancer Research Campaign (2001/2002) The challenge we face. In Scientific Yearbook, Berrington M (ed) pp 2-3. London: Park Communications

Coutinho L, Gilleece M, De Wynter E, Will A, Testa N (1993) Clonal and long-term cultures using human bone marrow. In Haemopoiesis: A Practical Approach, Testa NG, Molleneux G (eds) pp 75-106. Oxford UK: IRL Press

Chambers AF, Matrisian LM (1997) Changing views of the role of matrix metalloproteinases in metastasis. J Natl Cancer Inst 89: 1260 - 1270

Festuccia C, Dolo V, Guerra F, Violini S, Muzi P, Pavan A, Bologna M (1998) Plasminogen activator system modulates invasive capacity and proliferation in prostatic tumor cells. Clin Exp Metastasis 16: 513-528

George NJ (1988) Conservative management of prostate cancer [letter]. Lancet 1: 1001

Giambernardi TA, Grant GM, Taylor GP, Hay RJ, Maher VM, McCormick JJ, Klebe RJ (1998) Overview of matrix metalloproteinase expression in cultured human cells. Matrix Biol 16: 483-496

Hashimoto K, Kihira Y, Matuo Y, Usui T (1998) Expression of matrix metalloproteinase-7 and tissue inhibitor of metalloproteinase-1 in human prostate. J Urol 160: $1872-1876$

Heppner KJ, Matrisian LM, Jensen RA, Rodgers WH (1996) Expression of most matrix metalloproteinase family members in breast cancer represents a tumor-induced host response. Am J Pathol 149: 273-282

Hoosein N, Boyd D, Hollas W, Mazar A, Henkin J, Chung L (1991) Involvement of urokinase and its receptor in the invasiveness of human prostatic carcinoma cell lines. Cancer Communications 8: 255-264

Jacobs SC (1983) Spread of prostatic cancer to bone. Urology 21: 337-344

John A, Tuszynski G (2001) The role of matrix metalloproteinases in tumor angiogenesis and tumor metastasis. Pathol Oncol Res 7: 14-23

Kaighn ME, Narayan KS, Ohnuki Y, Lechner JF, Jones LW (1979) Establishment and characterization of a human prostatic carcinoma cell line (PC-3). Invest Urol 17: 16-23

Kirchheimer J, Pfluger H, Ritschl P, Heinert G, Binder B (1985) Plasminogen activator activity in bone marrow metastases of prostatic carcinomas as compared to primary tumors. Invasion Metastases 5: 344-355 prostate cell line PNT2-C2 when grown within BMS. We have demonstrated similar staining results using human prostate bone marrow metastases sections in vivo confirming the notion that in vivo observations reflect events observed in vitro. Inhibiting these enzymes reduced prostatic colony growth in the BMS, a finding supporting the proposition that these enzymes are important in development of metastases in humans. Our understanding of the metastatic process regarding MMP involvement is still limited, but clearly these enzymes play an important role in the invasion of bone marrow stroma by prostatic epithelial cells and bone marrow metastasis formation in man.

\section{ACKNOWLEDGEMENTS}

This work was supported by the Christie Hospital Surgical Research Fund and Cancer Research UK. SH Lang was funded by Yorkshire Cancer Research. The authors would like to thank pathologist Jonathan Shanks for the histological identification of all prostate samples used in this study.
Lang SH, Clarke NW, George NJ, Allen TD, Testa NG (1998) Interaction of prostate epithelial cells from benign and malignant tumor tissue with bone-marrow stroma. Prostate 34: 203-213

Lang SH, Stower M, Maitland NJ (2000) In vitro modelling of epithelial and stromal interactions in non-malignant and malignant prostates. $\mathrm{Br}$ Cancer 82: $990-997$

Lein M, Nowak L, Jung K, Koenig F, Schnorr D, Loening SA (1998) Metalloproteinases (MMP-1, MMP-3) and their inhibitors (TIMP) in blood plasma of patients with prostate carcinoma. Urologe A 37: 377-381

Marchenko GN, Ratnikov BI, Rozanov DV, Godzik A, Deryugina EI, Strongin AY (2001) Characterization of matrix metalloproteinase-26, a novel metalloproteinase widely expressed in cancer cells of epithelial origin. Biochem J 356: $705-718$

McIntyre IG, Spreckley K, Clarke RB, Anderson E, Clarke NW, George NJ (2000) Optimisation of the reverse transcriptase polymerase chain reaction for the detection of circulating prostate cells. Br J Cancer 83(8): 992 - 997

McIntyre I, Spreckley K, Clarke R, Anderson E, Clarke NW, George NJ (2001) Molecular prediction of progression of prostate cancer in conservatively managed patients. Urology 58(5): $762-766$

Migita T, Sato E, Saito K, Mizoi T, Shiiba K, Matsuno S, Nagura H, Ohtani H (1999) Differing expression of MMPs-1 and -9 and urokinase receptor between diffuse- and intestinal-type gastric carcinoma. Int J Cancer 84: $74-79$

Miyake H, Hara I, Yamanaka K, Gohji K, Arakawa S, Kamidono S (1999) Elevation of serum levels of urokinase-type plasminogen activator and its receptor is associated with disease progression and prognosis in patients with prostate cancer. Prostate 39: 123-129

Pajouh MS, Nagle RB, Breathnach R, Finch JS, Brawer MK, Bowden GT (1991) Expression of metalloproteinase genes in human prostate cancer. J Cancer Res Clin Oncol 117: 144-150

Saad S, Bendall LJ, James A, Gottlieb DJ, Bradstock KF (2000) Induction of matrix metalloproteinases MMP-1 and MMP-2 by co-culture of breast cancer cells and bone marrow fibroblasts. Breast Cancer Res Treat 63 $105-115$

Stetler-Stevenson WG, Yu AE (2001) Proteases in invasion: matrix metalloproteinases. Semin Cancer Biol 11: 143-152

Takahashi GW, Moran D, Andrews III DF, Singer JW (1994) Differential expression of collagenase by human fibroblasts and bone marrow stromal cells. Leukemia 8: $305-308$

Varani J, Hattori Y, Dame MK, Schmidt T, Murphy HS, Johnson KJ, Wojno KJ (2001) Matrix metalloproteinases (MMPs) in fresh human prostate tumour tissue and organ-cultured prostate tissue: levels of collagenolytic and gelatinolytic MMPs are low, variable and different in fresh tissue versus organ-cultured tissue. Br J Cancer 84: 1076-1083

Woolley DE (1993) Tumour cell growth and metastatic spread: an introductory overview. In Advances in the Biosciences, Vol 89, pp 1-29. Great Britain: Pergamon Press Ltd. 